\title{
Leverage for research in Africa
}

The editorial of March 2008 was inspired by a workshop in Yaounde, Cameroon, on the promotion of fruits and vegetables in the French-speaking countries of sub-Saharan Africa and the Indian Ocean. The aim was to mobilize around concrete actions not only the political representatives of these countries, but also the producers and other actors of the fruit and vegetable chain, representatives of research, health and education and, of course, funding agencies.

Since then some of these African countries have engaged in actions based on the recommendations of this workshop. Madagascar is one of them and, in this line, organized a national week on fruit production from 7 to 12 June 2010 and, a short time after, a national day on nutrition on the topic "the promotion of local products for good nutrition". These are encouraging signs that things are moving in the right direction despite a difficult context, and all efforts we can make to support such actions may have strong leverage to help these countries overcome their difficulties.

In another area, the Fruits journal, thanks to the motivation of its editor, was recently engaged in a training session in Chad, which aimed to strengthen the capacity of researchers to write scientific articles in international journals. It is clear that difficulties in scientific writing are a major obstacle to communicating and interacting with the international community, including English, and it is obvious that such training is again a leverage to help African scientists out of their isolation.

These two examples are further evidence that research findings do not only have an impact on themselves, but they must be integrated into more comprehensive measures, including training, awareness, advocacy and the participation of a wide range of stakeholders inside and outside the research community. With such an interplay between research and the society as a whole, a high leverage could be expected, especially in the poorest countries.

Dr. Jacky Ganry Chairman of the Section on Tropical and Subtropical Fruits

ISHS 


\section{Effet de levier de la recherche en Afrique}

L'éditorial du mois de mars 2008 avait été inspiré par un atelier à Yaoundé, Cameroun, portant sur la promotion des fruits et légumes dans les pays francophones de l'Afrique subsaharienne et l'Océan Indien. L'objectif était de mobiliser autour d'actions concrètes non seulement les représentants politiques de ces pays, mais aussi les producteurs et autres acteurs des filières fruits et légumes, les représentants de la recherche, de la santé et de l'éducation et, bien sûr, les organismes de financement.

Depuis lors, certains de ces pays africains se sont engagés dans des actions fondées sur les recommandations de cet atelier. Madagascar fait partie de ceux-ci et, dans cette ligne, a organisé une semaine nationale sur la production de fruits du 7 au 12 juin derniers, et, quelques jours après, une journée nationale sur la nutrition portant sur le thème de "la valorisation des produits locaux pour une bonne nutrition ". Ce sont des signes encourageants qui montrent que les choses bougent dans la bonne direction en dépit d'un contexte difficile et tous les efforts que nous pouvons faire pour soutenir de telles actions peuvent avoir de forts effets de levier pour aider ces pays à surmonter leurs difficultés.

Dans un autre domaine, la revue Fruits, grâce à la motivation de sa rédactrice en chef, a récemment participé à une séance de formation au Tchad, qui visait à renforcer les capacités des chercheurs pour la rédaction d'articles scientifiques dans des revues internationales. Il en ressort clairement que les difficultés dans la rédaction scientifique sont un obstacle majeur pour communiquer et interagir avec la communauté internationale, notamment anglophone, et il est évident qu'une telle formation aura là aussi un fort effet de levier pour aider les chercheurs africains à sortir de leur isolement.

Ces deux exemples sont une preuve supplémentaire que les résultats de recherches ne sont pas en mesure d'avoir un impact seulement par eux-mêmes, mais qu'ils doivent être intégrés dans des actions plus globales, comprenant la formation, la sensibilisation, le plaidoyer et la participation d'un large éventail d'acteurs faisant ou non partie de la communauté scientifique. Avec une telle osmose entre la recherche et la société dans son ensemble, un important effet de levier pourrait être espéré, tout particulièrement dans les pays les plus pauvres.

Dr. Jacky Ganry

Président de la section Fruits tropicaux et subtropicaux ISHS 